\title{
Decrease in intravenous thrombolysis and poor short-term functional prognosis for acute ischemic stroke during the COVID-19 pandemic
}

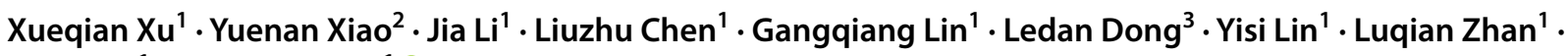 \\ Jincai He ${ }^{1}$ Xiaoqian Luan $^{1}$ (1)
}

Received: 24 July 2021 / Revised: 1 August 2021 / Accepted: 2 August 2021 / Published online: 11 August 2021

○) Springer-Verlag GmbH Germany, part of Springer Nature 2021

\begin{abstract}
Background and purpose Since the outbreak of the coronavirus pandemic in 2019 (COVID-19), healthcare systems around the world have been hit to varying degrees. As a neurologist team, for patients with acute ischemic stroke (AIS), we compared the situations of intravenous thrombolysis (IVT) treatment from 2019 to 2020 to investigate the influence of COVID-19 pandemic on the attendance and prognosis of the IVT patients.

Methods We collected the messages of objects who had received IVT (Bridging surgery was ruled out) during 2019-2020. We analyzed differences in age, gender, time from onset to start IVT, door to needle time (DNT), pretreatment NIHSS score, postoperative NIHSS score, and so on. Statistical tests were also performed to respectively compare the discharged modified Rankin score (mRS) and discharged NIHSS score between two years.

Results Since the onset of COVID-19 restrictions in Wenzhou, we observed a significant reduction of $24.7 \%(p=0.023)$ from 267(2019) to 201(2020) of received IVT on hospital admission. We compared the DNT between two years and it reflected that the DNT ( $\mathrm{min}$ ) in 2020 was obviously longer than in 2019 ( $51.60 \pm 23.80$ vs $46.80 \pm 21.90, p=0.026)$. We also compared the discharged mRS, which reflected much more IVT patients in 2020 during the COVID-19 pandemic had a poor short-term functional prognosis $(38.2 \%$ vs $29.2 \%, p=0.043)$.

Conclusions The COVID-19 pandemic caused the decrease of admissions and prolonged the time of the green channel for stroke, which led to the worse short-term prognosis of AIS patients during the pandemic. It's necessary to ensure an effective green channel and provide adequate medical resources during the pandemic period to reduce the damage caused by COVID-19.
\end{abstract}

Keywords COVID-19 Acute ischemic stroke $\cdot$ Intravenous thrombolysis $\cdot$ Functional prognosis $\cdot$ Door to needle time

Xueqian Xu, Yuenan Xiao, and Jia Li contributed equally to this work.

Jincai He

hjc@wmu.edu.cn

$\triangle$ Xiaoqian Luan

15058753868@163.com

1 Department of Neurology, The First Affiliated Hospital of Wenzhou Medical University, Wenzhou 325000, Zhejiang Province, China

2 Department of Neurosurgery, The First Affiliated Hospital of Wenzhou Medical University, Wenzhou 325000, China

3 Department of Radiology, The First Affiliated Hospital of Wenzhou Medical University, Wenzhou 325000, China

\section{Introduction}

Stroke is one of the common causes of mortality around the world, which also leads to a long-term neurological dysfunction [1]. Although its incidence, prevalence, mortality tend to decline from 1990 to 2013, the absolute number of people affected by stroke burden has increased around the world in both men and women of all age [2]. Intravenous thrombolysis (IVT) is a common treatment for acute ischemic stroke (AIS), which requires a highly organized approach to acute ischemic stroke treatment that enables patients to undergo life-saving procedures as soon as possible after acute ischemic stroke onset [3]. Usually, the total treatment from admission to discharge would work in a stroke unit. However, the outbreak of global coronavirus disease (COVID19) brought a big challenge to healthcare systems around 
the world, which also acted on stroke unit [4]. Recently, a research declared a decrease in hospital admissions for the transient ischemic attack, mild, and moderate stroke during the COVID-19 era [5]. A large study from Europe confirmed that intravenous thrombolytic treatments across Italy were reduced during the worst period of the COVID-19 outbreak [6].

Wenzhou is located in the southeast of China with a permanent population of 9.3 million. It was one of the areas most influenced by COVID-19, where more than 90,000 people came back from Wuhan days before the Spring Festival (February 12) [7]. Therefore, measures to prevent and control COVID-19 have resulted in a significant decrease in the efficiency of diagnosis and treatment in many hospitals in Wenzhou. As a neurologist team, we observed the diagnosis and treatment of patients with IVT treatment during the COVID-19 pandemic, the situations of the patients who received IVT treatment from 2019 to 2020 were compared. We developed this research to investigate the influence of the COVID-19 pandemic on the attendance and prognosis of IVT patients. It is hoped that this will help to treat IVT patients during the pandemic of COVID-19 in the future.

\section{Methods}

All data were obtained from the largest medical center of cerebrovascular disease in Wenzhou area (the first affiliated hospital of Wenzhou Medical University), where was equipped with an independent neuro-emergency clinic and mature Green Channel of AIS, where electronic medical records could be acquired. We collect the messages of the objects who had received IVT (Bridging surgery was ruled out) from 2019 to 2020. The steps of messages collecting were as follows: firstly, we derived data from the Green Channel of Stroke, which included the age, gender, time from onset to arrive hospital, time from onset to start IVT, door to needle time (DNT), pretreatment NIHSS score, postoperative NIHSS score and so on; Then, we collected the data such as discharged modified Rankin score (mRS), discharged NIHSS score in inpatient records of the neurology department. The NIHSS score has been divided in 4 parts $(0-4,5-8,9-14,>14)$ to get a better assessment of overall patient neurological function, the same classification as the Brazilian study. The short-term outcome was functional dependence or death when discharged from hospital defined as modified Rankin score (mRS) of 3-6, and the patients with a mRS score of 0-2 would be deemed to have a good prognosis [8].

The first confirmed case of COVID-19 in Wenzhou was in January 17, 2020. (Wenzhou Health Commission http://wjw. wenzhou.gov.cn/). On February 2, official decrees to start restrictions on social activities in Wenzhou were published.
(Wenzhou municipal people's government http://www. wenzhou.gov.cn). Until March 27, the strictest restrictions were lifted, but a series of restriction policies sustained for the whole year, especially after the rebound of the pandemic situation in June, so the daily activities of residents in the region in 2020 had significant changes from 2019, which also brought large changes in the medical system.

\section{Statistical analysis}

The continuous variables were presented as means \pm SD . Categorical variables were assessed by Pearson $\chi^{2}$ test. Statistical analysis was performed using SPSS Statistics 26.0 software. We used the line chart in Excel to show fluctuates of DNT or the number of IVT patients between 2019 and 2020. The bar chart in the SPSS was used to compare the short-term functional prognosis at discharge between the same months over two years. $p<0.05$ was considered statistically significant.

\section{Results}

The total number of patients with IVT was 201 in 2020, which is apparently less than the year 2019. We compared all stroke patients within the treatment time window and we found a lower proportion of IVT in 2020 than in 2019 ( $16.6 \%$ vs $24.6 \%$ ). Since the onset of COVID-19 restrictions in Wenzhou, we observed a significant reduction of $24.7 \%$ ( $p=0.023$ ) of received IVT on hospital admission, when compared with the total year in 2020 (Table 1). It showed that the inpatient number was decreased apparently

Table 1 Comparison of the number of people with thrombolysis therapy in different months (2019) before and (2020) after COVID-19

\begin{tabular}{llll}
\hline Months & $2019(n=267)$ & $2020(n=201)$ & $p$ \\
\hline Jan & 21 & 18 \\
Feb & 26 & 5 \\
Mar & 23 & 11 \\
Apr & 13 & 23 \\
May & 24 & 23 \\
Jun & 20 & 12 \\
Jul & 25 & 16 \\
Aug & 18 & 13 \\
Sep & 21 & 22 \\
Oct & 23 & 14 \\
Nov & 26 & 29 \\
Dec & 27 & 15
\end{tabular}

$0.023^{*}$

* $p$ values considered statistically significant 
in February and June in 2020 when compared with 2019, which was consistent with the time of restriction-policy be sustained (Fig. 1).

Futurely, we observed the difference in hospital admissions for AIS (2019) before and (2020) after COVID-19. Through the comparative analysis, it showed that there was no significant difference in the distribution of age $(p=0.218)$, gender $(p=0.170)$, time from onset to arrive hospital $(p=0.467)$ and time from onset to start IVT $(p=0.113$ ) between the two group. But when comparing the DNT between two years, it reflected that the DNT in 2020 was obviously longer than in $2019(51.60 \pm 23.80$ vs 46.80 $\pm 21.90, p=0.026$ ) (Table 2). The DNT in each month was shown that a significant difference was in July $(p=0.001)$, and also the same trend was seen in March, April, and June, which shows the great influence of the restriction policy on the hospital treatment process. And this trend disappeared in August which may owe to the effective control of the pandemic (Fig. 2).

Additionally, data presented the constitution of the patients had some difference. The pretreatment NIHSS score $(p=0.048)$ and the postoperative NIHSS score $(p=0.012)$ between the 2 years had significant variation, which showed the patients with a high NIHSS score have decreased from 2019 to 2020. And the difference of NIHSS between the postoperative and pretreatment showed that there was a poorer therapeutic effect in 2020 than 2019 (1.34 vs 0.52 . $(p=0.001))$ (Table 2). The discharged mRS reflected the much more IVT patients in 2020 have a poor short-term functional prognosis ( $38.2 \%$ vs $29.2 \%, p=0.043$ ), however, there was no difference in admission $\mathrm{mRS}(p=0.259)$, which concluded the same situation as the difference in NIHSS score between 2019 and 2020 that the pandemic might greatly affect the effectiveness of treatment. In Fig. 3, we had compared discharge mRS, which reflected Short-Term Functional Prognosis for AIS between corresponding months in a 2-year period. There was an interesting finding that longer DNT corresponded to poorer functional prognosis.

\section{Discussion}

As is known to all, early treatment of cerebral infarction plays an important role in the prognosis of patients. Also, appropriate treatment of ischemic stroke is essential in the reduction of mortality and morbidity. Management of stroke involves a multidisciplinary approach that starts and extends beyond hospital admission [9]. Alteplase significantly improved the overall odds of a good stroke outcome when delivered within $4.5 \mathrm{~h}$ of stroke onset, with earlier treatment associated with bigger proportional benefits [10]. In the present article, we provided the realworld data of the patients who received the IVT treatment during the COVID-19 pandemic in the whole year of 2020, compared with the same data in 2019. Firstly, we found that the number of patients receiving thrombolytic therapy decreased significantly in 2020 , which was in line with the decline in the number of patients seen by healthcare systems around the world in the face of the pandemic. A similar research in Brazil showed the number of patients admitted to hospital for stroke fell by $36.4 \%$ percent [5]. Another research in Italy also was confused with the baffling case of ischemic stroke disappearance from the casual department [11]. This situation appeared not only in neurology disease but also in other systems. A research in Austria showed the number of ST-segment elevation myocardial infarction (STEMI) patients reduced by $39.4 \%$ [12]. As the largest general hospital in Wenzhou, our research showed the number of patients admitted to the hospital for thrombolytic therapy decreased in most months. Due to the COVID-19 outbreak at the end of January, the number of patients had a precipitous fall. The same situation appeared in June, after the rebound of the pandemic. The restrictions on traffic might be an important factor that patients who lived in villages and towns around Wenzhou could arrive at the hospital formerly [13]. The restrictions increased the time for patients
Fig. 1 The trend of intravenous thrombolysis (2019) before and (2020) after COVID-19

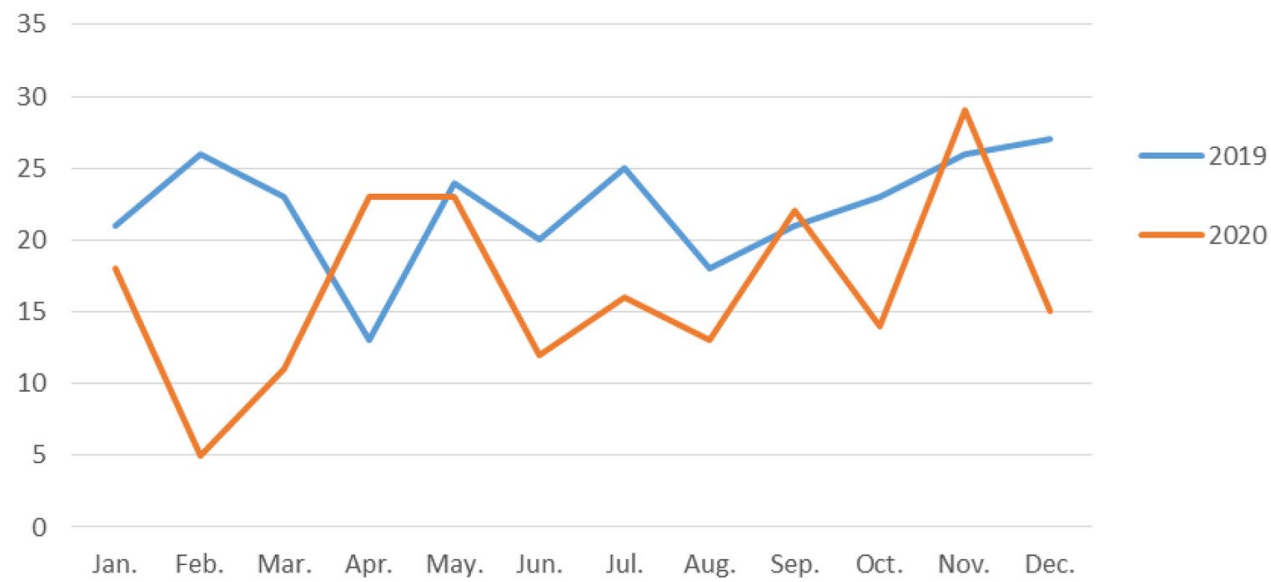


Table 2 Differences in hospital admissions for stroke (2019) before and (2020) after COVID19

\begin{tabular}{|c|c|c|c|}
\hline Parameters & $\begin{array}{l}2019 \\
(n=267)\end{array}$ & $\begin{array}{l}2020 \\
(n=201)\end{array}$ & $p$ \\
\hline Age,,$Y$ & $69.14 \pm 12.24$ & $67.72 \pm 12.35$ & 0.218 \\
\hline Gender (male, \%) & $55 \%$ & $61 \%$ & 0.170 \\
\hline Time from onset to arrive hospital (h) & $2.14 \pm 0.98$ & $2.21 \pm 1.15$ & 0.467 \\
\hline Time from onset to start IVT (h) & $2.92 \pm 0.99$ & $3.08 \pm 1.15$ & 0.113 \\
\hline DNT (min) & $46.80 \pm 21.90$ & $51.60 \pm 23.80$ & $0.026^{*}$ \\
\hline \multicolumn{4}{|l|}{ Pretreatment NIHSS (\%) } \\
\hline $0-4$ & 45.3 & 44.0 & \\
\hline $5-8$ & 17.6 & 28.0 & \\
\hline $9-14$ & 26.2 & 20.6 & \\
\hline$>14$ & 10.9 & 7.4 & \\
\hline Total & & & $0.048^{*}$ \\
\hline \multicolumn{4}{|l|}{ Postoperative NIHSS (\%) } \\
\hline $0-4$ & 56.3 & 48.0 & \\
\hline $5-8$ & 13.5 & 27.3 & \\
\hline $9-14$ & 21.1 & 16.7 & \\
\hline$>14$ & 9.1 & 8.0 & \\
\hline Total & & & $0.012 *$ \\
\hline Postoperative NIHSS—Pretreatment NIHSS & 1.34 & 0.52 & $0.001 * * *$ \\
\hline \multicolumn{4}{|l|}{ mRS (admission) (\%) } \\
\hline $0-2$ & 52.3 & 46.7 & \\
\hline $3-6$ & 47.7 & 53.3 & \\
\hline Total & & & 0.259 \\
\hline \multicolumn{4}{|l|}{ mRS (discharged) $(\%)$} \\
\hline $0-2$ & 70.8 & 61.8 & \\
\hline $3-6$ & 29.2 & 38.2 & \\
\hline Total & & & $0.043 *$ \\
\hline
\end{tabular}

IVT intravenous thrombolysis, DNT door to needle time, NIHSS The National Institutes of Health Stroke Scale, $m R S$ Modified Rankin Scale

${ }^{*} p$ values considered statistically significant; $* p<0.05, * * * p<=0.001$

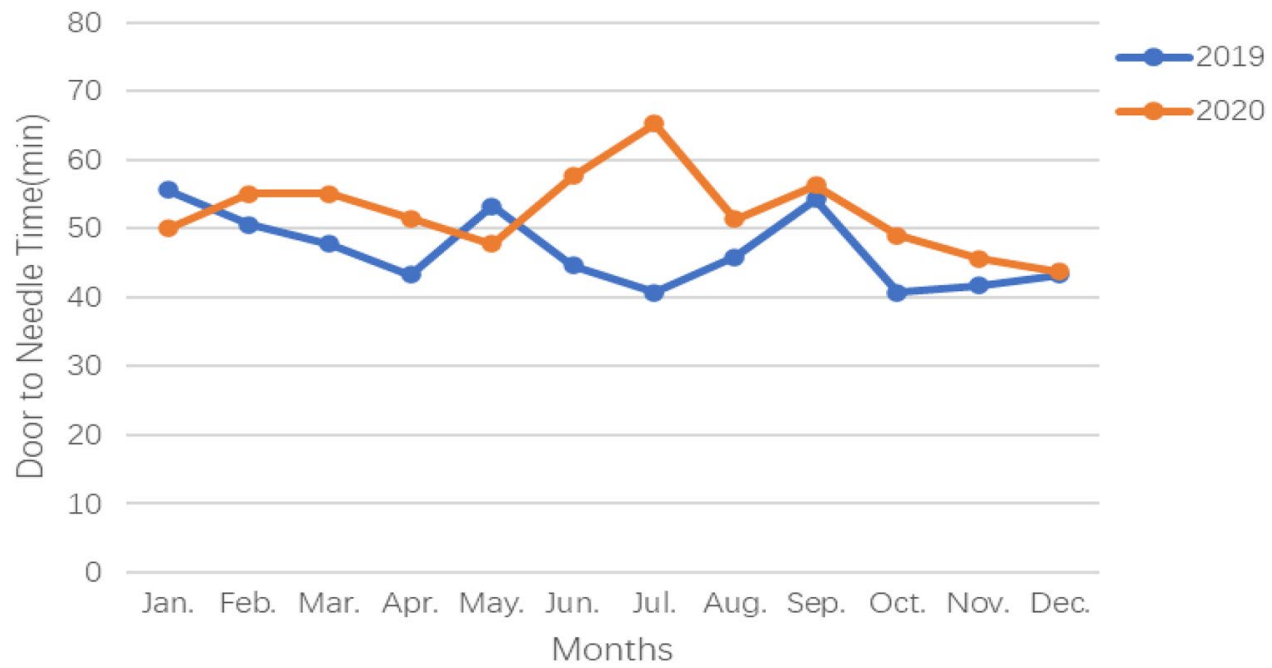


Fig. 3 The difference of shortterm functional prognosis between corresponding months (2019) before and (2020) after COVID-19

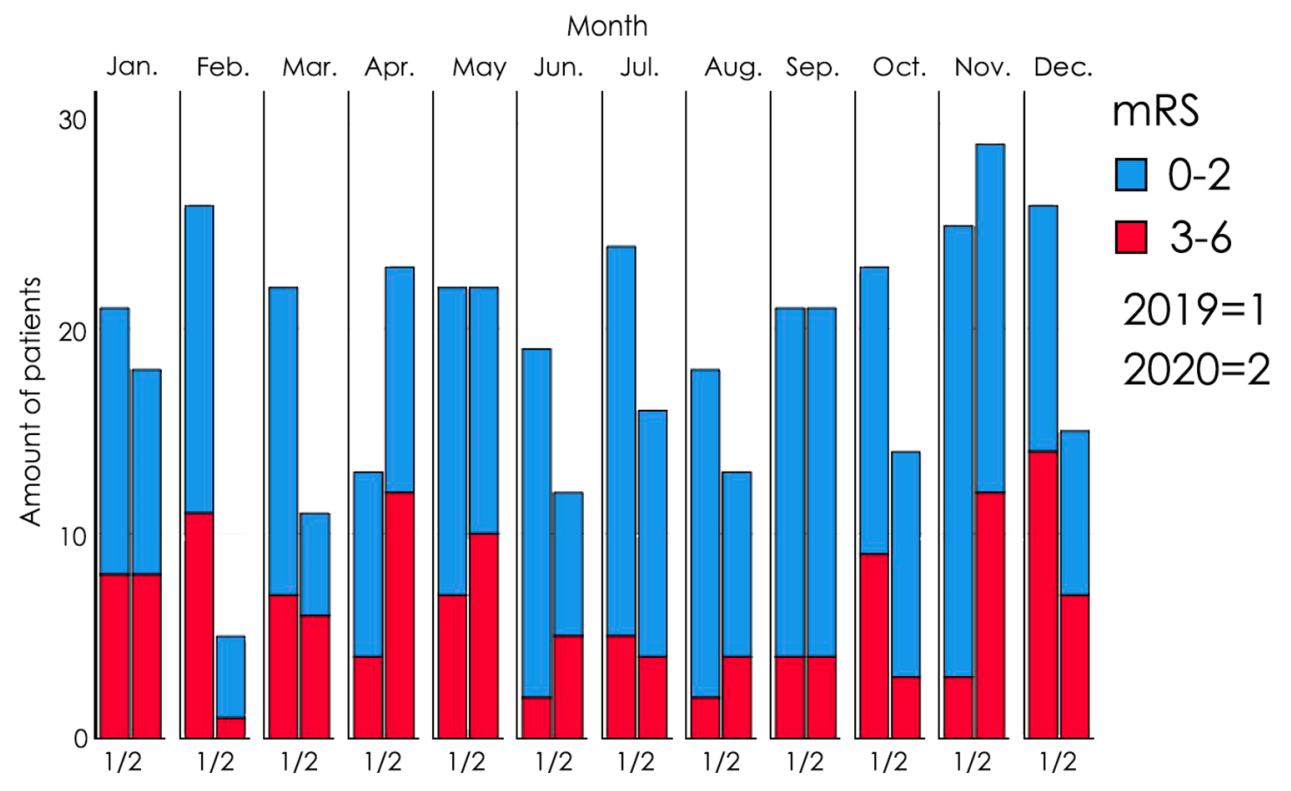

to arrive at a district medical center so that patients and their relatives were more likely to go to their local hospital [14]. Another reason was that more patients exceeding the treatment time window which decreased the number of patients who received treatment successfully. They could only receive conservative treatment because of this. To sum up, the pandemic did cause inconvenience for stroke patients. Therefore, it was necessary to establish modified stroke care networks and allocate resources efficiently to reduce the negative impact of COVID-19.

We also compared if there were any other differences between the two groups. Age, gender, and time from onset to arrive hospital or start IVT had no difference. But interestingly, we found that patients' DNT increased in 2020, which mean the patients were staying longer in the hospital before they received thrombolysis therapy. What's more, we observed the biggest differences DNT in February, June, and July, in line with the in-patient numbers. The main reason might be the pandemic delayed the green channel of stroke. In accordance with the Get With The Guidelines findings, interhospital transfers were also a significant predictor for an unfavorable outcome in the GSR-ET, even after adjustment for potential confounders including time from symptom onset to groin puncture [15], therefore the epidemic increased the time of hospital transfer. Considering that our hospital was the largest diagnosis and treatment center for cerebral infarction patients in the region, the COVID19 actually magnified the damaging effect of such hospital transfer. In accordance with the hospital's pandemic prevention regulations, all patients should have a coronavirus test, the CT machine should be disinfected after each patient has completed the CT examination. All of this could systematically slow down the rate at which patients receive treatment.
Another reason was that because of the pandemic, hospital staff began to work in shifts, and the reduction of staff has greatly slowed the flow of patients in the hospital. A joint research of more than 40 countries around the world shows that the center volume had a robust effect on DNT [16]. Pandemic had taken up too many medical resources and reduced the capacity of hospitals for stroke. How to balance the control of pandemic and efficiency of green channel would be an urgent problem for hospitals to solve.

The COVID-19 pandemic also affected to patients' outcome. The data showed the discharged mRS increased significantly in 2020. Since there was no difference in time from onset to arrive hospital or start IVT among hospitalized patients, we had reason to believe that the pandemic was mainly affected post-admission treatment procedures. One of the reasons might be the variation of DNT. One study in the Netherlands showed that early care helped reduce DNT time and decreased patients' NIHSS scores [17], and another study in the Netherlands showed that overwork affected DNT time [18]. Given that there was no difference in the time between onset and admission of patients in this study, the effect of DNT on outcomes may have been magnified, leading to worse outcomes in 2020 than in 2019. In addition, we compared NIHSS score differences (Postoperative NIHSSPretreatment NIHSS) over two years, showing differences consistent with mRS scores. This suggested that the quality of thrombolytic therapy had declined during the pandemic, and a study in Brussels concluded that out-of-hours thrombolytic therapy can lead to poorer outcomes, possibly due to doctors' long working hours and fatigue [19]. In Fig. 3, we had compared discharge mRS, which reflected ShortTerm Functional Prognosis for AIS between corresponding months in a two-year period. There was an interesting 
finding that longer DNT corresponded to poorer functional prognosis, which conformed to the view that among patients aged 65 years or older with acute ischemic stroke who were treated with tissue plasminogen activator, shorter door-toneedle times were associated with lower all-cause mortality and lower all-cause readmission at 1 year [20].

To sum up, the COVID-19 pandemic caused a certain impact on AIS treatment in Wenzhou, especially prolonged the time of green channel for stroke, which led to the worse prognosis of stroke patients during the pandemic. Therefore, ensuring an effective green channel during the pandemic period, providing adequate medical resources, and constructing a special framework that was convenient for implementation during the pandemic period will effectively help thrombolytic therapy during the pandemic period and improve the prognosis of patients [21]. With the people commonly vaccinated, a gradual easing of travel restrictions would greatly improve the prognosis of stroke patients. At the same time, restoring normal shifts for medical staff and hospital administrators as soon as possible would help reduce DNT.

Author contributions Authors Xiaoqian Luan, Jia Li, Jincai He designed the study and wrote the protocol. Authors Xueqian Xu, Yuenan, Xiao, Liuzhu Chen, Gangqiang Lin, Ledan Dong, Yisi Lin, Luqian Zhan conducted literature searches and provided summaries of previous research studies. Author XiaoqianLuan conducted the statistical analysis. Author Xueqian Xu wrote the first draft of the manuscript and all authors contributed to and have approved the final manuscript.

Funding This research was supported by a grant from Wenzhou Municipal Sci-Tech Bureau Program (Y2020421). We are greatly indebted to the staff and to the patients with stroke for their contributions during this study.

\section{Declarations}

Conflicts of interest All authors declare that they have no conflicts of interest.

Ethical approval This protocol was approved by the ethics committee of the First Affiliated Hospital of Wenzhou Medical University and was in accordance with the Declaration of Helsinki.

Informed consent Informed consent was obtained from each participant.

\section{References}

1. Feigin VL, Forouzanfar MH, Krishnamurthi R, Mensah GA, Connor M, Bennett DA et al (2014) Global and regional burden of stroke during 1990-2010: findings from the Global Burden of Disease Study 2010. Lancet 383(9913):245-254

2. Feigin VL, Norrving B, Mensah GA (2017) Global Burden of Stroke. Circ Res 120(3):439-448

3. Campbell BCV, De Silva DA, Macleod MR, Coutts SB, Schwamm LH, Davis SM et al (2019) Ischaemic stroke. Nat Rev Dis Primers 10:5

4. Zhao J, Li H, Kung D, Fisher M, Shen Y, Liu R (2020) Impact of the COVID-19 epidemic on stroke care and potential solutions. Stroke 51(7):1996-2001
5. Diegoli H, Magalhaes PSC, Martins SCO, Moro CHC, Franca PHC, Safanelli J et al (2020) Decrease in hospital admissions for transient ischemic attack, mild, and moderate stroke during the COVID-19 era. Stroke 51(8):2315-2321

6. Sacco S, Ricci S, Ornello R, Eusebi P, Petraglia L, Toni D (2020) Reduced admissions for cerebrovascular events during COVID-19 outbreak in Italy. Stroke 51(12):3746-3750

7. Ruan L, Wen M, Zeng Q, Chen C, Huang S, Yang S et al (2020) New measures for the Coronavirus Disease 2019 response: a lesson from the Wenzhou experience. Clin Infect Dis 71(15):866-869

8. Lux D, Alakbarzade V, Bridge L, Clark CN, Clarke B, Zhang L et al (2020) The association of neutrophil-lymphocyte ratio and lymphocyte-monocyte ratio with 3-month clinical outcome after mechanical thrombectomy following stroke. J Neuroinflamm 17(1):60

9. Herpich F, Rincon F (2020) Management of acute ischemic stroke. Crit Care Med 48(11):1654-1663

10. Emberson J, Lees KR, Lyden P, Blackwell L, Albers G, Bluhmki E et al (2014) Effect of treatment delay, age, and stroke severity on the effects of intravenous thrombolysis with alteplase for acute ischaemic stroke: a meta-analysis of individual patient data from randomised trials. Lancet 384(9958):1929-1935

11. Morelli N, Rota E, Terracciano C, Immovilli P, Spallazzi M, Colombi D et al (2020) The baffling case of ischemic stroke disappearance from the casualty department in the COVID-19 era. Eur Neurol 83(2):213-215

12. Metzler B, Siostrzonek P, Binder RK, Bauer A, Reinstadler SJ (2020) Decline of acute coronary syndrome admissions in Austria since the outbreak of COVID-19: the pandemic response causes cardiac collateral damage. Eur Heart J 41(19):1852-1853

13. Oum TH, Wang K (2020) Socially optimal lockdown and travel restrictions for fighting communicable virus including COVID-19. Transp Policy 96:94-100

14. Venema E, Burke JF, Roozenbeek B, Nelson J, Lingsma HF, Dippel DWJ et al (2020) Prehospital triage strategies for the transportation of suspected stroke patients in the United States. Stroke 51(11):3310-3319

15. Wollenweber FA, Tiedt S, Alegiani A, Alber B, Bangard C, Berrouschot J et al (2019) Functional outcome following stroke thrombectomy in clinical practice. Stroke 50(9):2500-2506

16. Strbian D, Ahmed N, Wahlgren N, Lees KR, Toni D, Roffe C et al (2015) Trends in door-to-thrombolysis time in the safe implementation of stroke thrombolysis registry: effect of center volume and duration of registry membership. Stroke 46(5):1275-1280

17. Zinkstok SM, Beenen LF, Luitse JS, Majoie CB, Nederkoorn PJ, Roos YB (2016) Thrombolysis in stroke within 30 minutes: results of the acute brain care intervention study. PLoS ONE 11(11): 0166668

18. Groot AE, de Bruin H, Nguyen TTM, Kappelhof M, de Beer F, Visser MC et al (2021) Presentation outside office hours does not negatively influence treatment times for reperfusion therapy for acute ischemic stroke. J Neurol 268(1):133-139

19. Hoepner R, Weber R, Reimann G, Berger K, Kitzrow M, Fischer S et al (2019) Stroke admission outside daytime working hours delays mechanical thrombectomy and worsens short-term outcome. Int J Stroke 14(5):517-521

20. Man SXY, Holmes DN, Matsouaka RA, Saver JL, Smith EE, Bhatt DL, Schwamm LH, Fonarow GC (2020) Association between thrombolytic door-to-needle time and 1-year mortality and readmission in patients with acute ischemic stroke. JAMA 323(21):2170-2184

21. Khosravani H, Rajendram P, Notario L, Chapman MG, Menon BK (2020) Protected code stroke: hyperacute stroke management during the Coronavirus Disease 2019 (COVID-19) pandemic. Stroke 51(6):1891-1895 